\title{
The Localization Length of Stationary States in the Nonlinear Schrödinger Equation
}

\author{
Alexander Iomin and Shmuel Fishman \\ Department of Physics, Technion, Haifa, 32000, Israel
}

(Dated: August 14, 2021)

\begin{abstract}
For the nonlinear Schrödinger equation (NLSE), in presence of disorder, exponentially localized stationary states are found. In the present work it is demonstrated analytically that the localization length is typically independent of the strength of the nonlinearity and is identical to the one found for the corresponding linear equation. The analysis makes use of the correspondence between the stationary NLSE and the Langevin equation as well as of the resulting Fokker-Planck equation. The calculations are performed for the "white noise" random potential and an exact expression for the exponential growth of the eigenstates is obtained analytically. It is argued that the main conclusions are robust.
\end{abstract}

PACS numbers: 72.15.Rn, 42.25.Dd, 42.65.k 
In this work we consider a simple problem of the one-dimensional Anderson localization [1, 2] for the nonlinear Schrödinger equation (NLSE) [3, 4, 5]. This problem is relevant to experiments in nonlinear optics, for example disordered photonic lattices [6], where Anderson localization was found in presence of nonlinear effects as well as experiments on Bose-Einstein Condensates (BEC) in disordered optical lattices [7, 8, 9, 10, 11]. The interplay between disorder and nonlinear effects leads to new interesting physics [8, 9, 12, 13, 14, 15]. In particular, the problem of spreading of wave packets and transmission are not simply related [16, 17], in contrast with the linear case. In spite of the extensive research, many fundamental problems are still open, and, in particular, it is not clear whether in one dimension (1D) Anderson localization can survive the effects of nonlinearities.

Herein we consider 1D localization of stationary solutions of the NLSE in a random potential. The problem is described by the equation

$$
i \partial_{t} \psi=-\partial_{x}^{2} \psi+\beta|\psi|^{2} \psi+V(x) \psi
$$

where $V(x)$ is a random $\delta$-correlated potential with a Gaussian distribution, of zero mean and variance $\sigma^{2}$, such that

$$
\left\langle V(x) V\left(x^{\prime}\right)\right\rangle=2 \sigma^{2} \delta\left(x-x^{\prime}\right)
$$

where $\langle\ldots\rangle$ denotes the average over realizations of the random potential. The variables are chosen in dimensionless units and the Planck constant is $\hbar=1$. For the linear case $(\beta=0)$ this model was studied extensively in the past [18, 19]. The problem in question is Anderson localization of stationary solutions of Eq. (1) with energies $\omega$

$$
\psi(x, t)=\exp (-i \omega t) \phi(x)
$$

where $\phi(x)$ is real. Substituting Eq. (3) in Eq. (1) one obtains the stationary NLSE

$$
\omega \phi(x)=-\partial_{x}^{2} \phi(x)+\beta \phi^{3}(x)+V(x) \phi(x) .
$$

It was established rigorously [3, 20, 21] that this equation has exponentially localized solutions of this type for a wide range conditions. It is instructive to notice that also in absence of disorder $(V=0)$ the nonlinear equation exhibits stationary localized states, and these are not simply related to the localized states in presence of disorder [13, 14, 22]. The purpose of the present work is to find the localization length of these states. It will turn out that the 
localization length is typically not affected by the nonlinearity and is identical to the one of the linear problem $(\beta=0)$. The approach a la Borland [23, 24] will be the basis of our analysis. This approach is reviewed clearly in detail in [25] and made rigorous in [26].

We will specifically calculate $\left\langle\phi^{2}(x)\right\rangle$ of solutions of Eq. (4) that are found for a certain $\omega$, with given boundary conditions at some point, for example $\phi(x=0)$ and $\phi^{\prime}(x=0)$, where prime means the derivative with respect to $x$. It will be shown that this quantity grows exponentially with the rate

$$
2 \gamma=\lim _{x \rightarrow \infty} \frac{\ln \left\langle\phi^{2}(x)\right\rangle}{x}>0, \quad \xi=\frac{1}{\gamma},
$$

that is independent of $\beta$, where $\xi$ is the localization length. Note, it is different from the usually studied self averaging quantity $\gamma_{s}=\frac{1}{2} \frac{d}{d x}\left\langle\ln \phi^{2}(x)\right\rangle=\frac{1}{2} \lim _{x \rightarrow \infty} \frac{\ln \phi^{2}(x)}{x}$. We will find that $\gamma$ is a smooth function of energy. Since the distribution of random potentials is translationally invariant, it is independent of the choice of the initial point as $x=0$. Like in the linear case, starting from a specific initial condition, $\phi(x)$ will typically grow. For specific values of $\omega$ at some point this function will start to decay, so that a normalized eigenfunction is found [23, 24, 25, 26]. The envelope of the wave function will grow exponentially if we start either from the right or from the left. The value of $\omega$ results from the matching condition, so that an eigenfunction has some maximum and decays in both directions as required by the normalization condition. The exponential decay is an asymptotic property, while the matching is determined by the potential in the vicinity of the maximum. This observation [25, 26] is crucial for the validity of this approach and enables us to determine the exponential decay rate of states from the solution of the initial value problem (4). For the linear case these values of $\omega$ form the point spectrum of the problem that is the entire spectrum of the linear problem. This approach can be followed also for the nonlinear problem, but contrary to the linear case, these stationary states do not provide a complete picture of the dynamics. Let us fix $\omega$, the stationary states in the vicinity of $\omega$ will on the average (over realizations) decay with the localization length $\xi$ defined by Eq. (5). As mentioned it will be found to be independent of $\beta$, leading to the conclusion that this is a typical property of the localized eigenstates of Eq. (4).

The calculation of $\left\langle\phi^{2}(x)\right\rangle$ will be performed by the analogy with the classical Langevin equation [18, 19]. Therefore, here we are considering the $x$-coordinate as the formal time on 
the half axis $x \equiv \tau \in[0, \infty)$, and Eq. (44) reduces to the Langevin equation

$$
\ddot{\phi}+\omega \phi-\beta \phi^{3}-V(\tau) \phi=0
$$

with the $\delta$ correlated Gaussian noise $V(\tau)$. Now we introduce new variables $u=\phi$ and $v=\dot{\phi} \equiv \frac{d \phi}{d \tau}$ and a distribution function of these new variables is $P=P(u, v, \tau)$. The dynamical process in the presence of the Gaussian $\delta$-correlated noise is described by the distribution function that satisfies the Fokker-Planck equation: (FPE) (see, e.g., [27, 28])

$$
\partial_{\tau} P-\left[\omega u-\beta u^{3}\right] \partial_{v} P+v \partial_{u} P-\sigma^{2} u^{2} \partial_{v}^{2} P=0
$$

It is obtained from the Langevin equation (6), as can be seen in [27, 28] and is consistent with [19], Eq. (6.14) there [29].

We are interested in the average quantum probability density $\left\langle\phi^{2}(x)\right\rangle \equiv\left\langle u^{2}(\tau)\right\rangle$, where

$$
\left\langle u^{2}(\tau)\right\rangle=\int u^{2} P(u, v, \tau) d u d v
$$

It is useful to obtain from the FPE a system of equations for the moments

$$
M_{k, l}=\left\langle u^{k} v^{l}\right\rangle
$$

where $k, l=0,1,2, \ldots$. Substituting $u^{k} v^{l}$ in the FPE and integrating over $u$ and $v$, one obtains the following relation for $M_{k, l}$

$$
\dot{M}_{k, l}=-l \omega M_{k+1, l-1}+k M_{k-1, l+1}+l(l-1) \sigma^{2} M_{k+2, l-2}+\beta l M_{k+3, l-1},
$$

where $M_{k, l}$ with negative indexes are assumed to vanish. We note that only terms with the same parity of $k+l$ are coupled. Since we are interested in $M_{2,0}=\left\langle u^{2}\right\rangle$, we study only the case when this parity is even, namely $k+l=2 n$ with $n=1,2, \ldots$. The sum of the indexes of the moments is $2 n$, except the last term $\beta l M_{k+3, l-1}$, where the sum is $2(n+1)$. This leads to the infinite system of linear equations that can be written in the form

$$
\dot{\mathbf{M}}=\mathcal{W} \mathbf{M}
$$

where $\mathbf{M}=\left(M_{2,0}, M_{1,1}, M_{0,2}, M_{4,0}, M_{3,1}, \ldots\right)$ and $=\mathcal{W}$ is the corresponding matrix. The matrix elements $\mathcal{W}_{k, l}$ are determined by Eq. (9). The solutions of the system of linear equations (10) are linear combinations of the eigenfunctions

$$
M_{\lambda}(t)=\exp (\lambda \tau) M_{\lambda}(0)
$$


where $M_{\lambda}(0)$ is the eigenvector of $\mathcal{W}$ corresponding to $\lambda$. The growth rate of each moment, in particular $M_{2,0}=\left\langle u^{2}\right\rangle$, is determined by the eigenvalue with the largest real part $\operatorname{Re} \lambda$.

For $\beta=0$, Eq. (9) (or (10)) has a closed form for each $n$. Therefore, the infinite matrix $\mathcal{W}$ is block diagonal and consists of the independent blocks $A_{n}=A_{n}[(2 n+1) \times(2 n+1)]$, and the characteristic polynomial reduces to a product of their determinants

$$
\prod_{n=1}^{\infty} \operatorname{det}\left(A_{n}-\lambda I_{n}\right)=0
$$

where $I_{n}$ is an $(2 n+1) \times(2 n+1)$ unit matrix. The problem of localization in the framework of Eq. (9) reduces to $n=1$. The relevant characteristic polynomial $\operatorname{det}\left(A_{1}-\lambda I_{1}\right)$ reduces to a cubic equation

$$
\lambda^{3}+4 \omega \lambda-4 \sigma^{2}=0 .
$$

Cardano's method yields [30]

$$
\lambda_{1}=R_{+}+R_{-}, \quad \lambda_{2,3}=-\frac{R_{+}+R_{-}}{2} \pm i \sqrt{3} \frac{R_{+}-R_{-}}{2}
$$

where $R_{ \pm}=\left[2 \sigma^{2} \pm \sqrt{4 \sigma^{4}+\frac{64 \omega^{3}}{27}}\right]^{\frac{1}{3}}$. The growth rate is determined by the eigenvalue with largest real part that will be denoted by $\lambda_{m}$. We conclude that asymptotically for large $x$ the averaged wave function indeed grows exponentially as $\left\langle\phi^{2}(x)\right\rangle \sim e^{2 \gamma x}$ and $2 \gamma=\lambda_{m}$. Also the behavior of the higher blocks can be calculated. From the $n$-th block the behavior of the moment $\left\langle\phi^{2 n}(x)\right\rangle$ can be found. In the high energy limit one finds from Eq. (14)

$$
\begin{gathered}
\lambda_{m}=\lambda_{1} \approx \frac{\sigma^{2}}{\omega}, \quad \omega \rightarrow+\infty, \\
\lambda_{m}=\operatorname{Re} \lambda_{3} \approx 2 \sqrt{|\omega|}, \quad \omega \rightarrow-\infty .
\end{gathered}
$$

These limits can be found directly from Eq. (13). The solutions should be compared with the high energy asymptotics obtained in Ref [19] (Eq. (10.12), p. 143), where $\gamma_{s}=\sigma^{2} / 4 \omega$, and $\gamma_{s}=\sqrt{|\omega|}$ in limits $\omega \rightarrow+\infty$ and $\omega \rightarrow-\infty$, respectively. Note that Eq. (14) gives a simple expression for $\gamma$ for all values of the parameters, while the expression for $\gamma_{s}$ is known only in the large $\omega$ limit. Since $\gamma$ and $\gamma_{s}$ result of different averages, these are not expected to be identical.

Now, let us consider localization for $\beta \neq 0$. The eigenvalue equation

$$
\mathcal{W}(\beta) M_{\lambda}(\beta)=\lambda(\beta) M_{\lambda}(\beta)
$$


is obtained from Eq. (9), $\mathcal{W}$ is not block diagonal anymore. The $\beta$ dependence results from the last term in Eq. (9). The $\beta$ dependent terms couple the $n$-th block and $(n+1)$ block and are located above the $(n+1)$ block and to the right of the $n$-th block. Consequently, the $\beta$ dependent terms do not affect the characteristic polynomial, as can be shown by elementary operations on determinants. It reduces to the one found for $\beta=0$, namely Eq. (12). Therefore, growth rates of all moments of $\phi(x)$ do not depend on $\beta$ and their values are equal to the ones of the linear problem for $\beta=0$. This is correct in particular for $\lambda_{m}$, consequently $\gamma$ of Eq. (5) is identical to the value found in the linear case $(\beta=0)$.

We demonstrated that in the presence of a random potential the stationary states of the NLSE are exponentially localized with the localization length that is found in the absence of the nonlinearity. This is in agreement with a heuristic argument that the effect of nonlinearity is negligible where the wave function is small. We believe that the approach á la Borland can be extended to a rigorous treatment of the stationary states of the NLSE. Since this equation is nonlinear, the stationary states do not provide the complete or even essential description of the dynamics, starting from a given initial condition. The status of stability of these states with respect of small perturbations is not clear. The relation to the transmission problem is not obvious. Our results are consistent with the limit of vanishing flux in the transmission problem [31].

Another question that should be discussed is of the generality of the results. Assume that the power of $\psi$ in Eq. (11) differs from 3. Only the last term in Eq. (91) will be affected resulting in a different coupling between the blocks of the matrix $\mathcal{W}$. But since these couplings are above the diagonal of the block diagonal matrix, they will not affect our conclusion that the characteristic polynomial which determines $\lambda$ is not affected by the nonlinearity. If the potential $V(x)$ deviates from a white noise one, Kramers-Moyal coefficients [27] that are higher than the second one appear. But if no convergence problems of the Kramers-Moyal expansion are encountered, an equation like Eq. (77) with higher powers of $u$ and $v$, combined with higher order derivatives is obtained. Because of the structure of the Kramers-Moyal expansion the block diagonal form of the matrix $\mathcal{W}$ in absence of the nonlinearity is expected to be unaffected by this deviation from white noise. Therefore we expect the main result of the work, namely the independence of the localization length of the nonlinearity to exhibit some degree of robustness and to hold for a wide range of models, beyond the specific model that is studied in detail in the present work. All these 
problems should be subject of further studies.

This work was supported in part by the Israel Science Foundation (ISF), by the US-Israel Binational Science Foundation (BSF), and by the Minerva Center for Nonlinear Physics of Complex Systems. It is our great please to thank I. Guarneri and A. Soffer for very critical discussions that affected some directions of our work and E. Baskin, S. Flach, Y. Krivolapov, B. Shapiro, and D. Shepelyansky for very informative and instructive discussions and communications. We thank S. Aubry, S. Gredeskul, P. Leboeuf, and N. Pavloff for communications and comments after the work was completed.

[1] P.W. Anderson, Phys. Rev. 109, 1492 (1958).

[2] P. A. Lee and T. V. Ramakrishnan, Rev. Mod. Phys. 57, 287 (1985).

[3] J. Fröhlich, T. Spencer, and C.E. Wayne, J. Stat. Phys. 432, 247 (1986).

[4] P. Devillard and B.J. Souillard, J. Stat. Phys. 43, 423 (1986).

[5] S.A. Gredeskul and Y.S. Kivshar, Phys. Rep. 216, 1 (1992).

[6] T. Schwartz, G. Bartal, S. Fishman, and M. Segev, Nature 446, 52 (2007), and references therein.

[7] H. Gimperlein, S. Wessel, J. Schmiedmayer, and L. Santos, Phys. Rev. Lett. 95, 170401 (2005); J. E. Lye, et al., Phys. Rev. Lett. 95, 070401 (2005); Clement, et al., Phys. Rev. Lett. 95, 170409 (2005).

[8] C. Fort, et al., Phys. Rev. Lett. 95, 170410 (2005).

[9] E. Akkermans, S. Ghosh, and Z. Musslimani, Numerical study of one-dimensional and interacting Bose-Einstein condensates in a random potential, cond-mat/0610579.

[10] L. Sanchez-Palencia, et al., Phys. Rev. Lett. 98, 210401 (2007).

[11] B. Shapiro, Expansion of a Bose-Einstein Condensate in the Presence of Disorder, cond-mat/0701346.

[12] A.R. Bishop (editor) Fluctuation Phenomena: Disorder and Nonlinearity (World Scientific Publishing Company, 1995); K.O. Rasmussen, D. Cai, A.R. Bishop, and N. Gronbech-Jensen, Europhys. Lett. 47, 421 (1999); D.K. Campbell, S. Flach, and Y.S. Kivshar, Physics Today 57, 43 (2004); D.L. Shepelyansky, Phys. Rev. Lett. 70, 1787 (1993); M.I. Molina, Phys. Rev. B 58, 12547 (1998). 
[13] G. Kopidakis and S. Aubry, Phys. Rev. Lett. 84, 3226 (2000).

[14] R.S. MacKay and S. Aubry, Nonlinearity bf 7, 1623 (1994).

[15] G. Kopidakis and S. Aubry, Physica D 130, 155 (1999); 139, 247 (2000).

[16] B. Doucot and R. Rammal, Europhys. Lett. 3, 969 (1987); J. Physique (Paris) 48, 527 (1987).

[17] T. Paul, P. Schlagheck, P. Leboeuf, and N. Pavloff, Phys. Rev. Lett. 98, 210602 (2007).

[18] B.I. Halperin, Phys. Rev. 139A, 104 (1965).

[19] I.M. Lifshits, S.A. Gredeskul, and L.A. Pastur, Introduction to the theory of disordered systems (Wiley-Interscience, New York, 1988).

[20] C. Albanese and J. Fröhlich, Comm. Math. Phys. 116, 475 (1988).

[21] C. Albanese, J. Fröhlich, and T. Spencer, Comm. Math. Phys. 119, 677 (1988); C. Albanese and J. Fröhlich, Comm. Math. Phys. 138, 193 (1991).

[22] S. Flach, Phys. Rev. E 50, 3134 (1994); Phys. Rev. E 58, R4116 (1998); A.V. Gorbach and S. Flach, Phys. Rev. E 72, 056607 (2005); B. Day, M. Eleftheriou, S. Flach and G.P. Tsironis, Phys. Rev. E 65, 017601 (2001).

[23] R.E. Borland, Poc. Roy. Soc.274, 529 (1963).

[24] N.M. Mott and W.D. Twose, Adv. Phys. 10, 107 (1961); N.F. Mott, Adv. Phys. 1649 (1967).

[25] F. Delyon, Y. Levy and B. Souillard, Phys. Rev. Lett. 55, 618 (1985).

[26] F. Delyon, Y. Levy and B. Souillard, J. Stat. Phys. 41, 375 (1985); Commun. Math. Phys. 100, 463 (1985).

[27] H. Risken, The Fokker-Planck equation (Springer-Verlag, Berlin, 1989).

[28] S.M. Rytov, Yu.A. Kravtsov, and V.I. Tatarskii, Principles of statistical radiophysics (Springer, Berlin, 1987).

[29] To see this one has to define $P(z, \tau)=\int d u d v \delta\left(z-\frac{v}{u}\right) P(u, v, \tau)$ and derive Eq. (6.14) of [19] from Eq. (7).

[30] G.A. Korn and T.M. Korn, Mathematical handbook for scientists and engineers (McGraw-Hill, NY, 1968).

[31] N. Pavloff and P. Leboeuf, Private communication and Ref. 17]. 